Forum 2021 · 36:269-271

https://doi.org/10.1007/s12312-021-00954-9

Online publiziert: 13. Juli 2021

(c) Springer Medizin Verlag $\mathrm{GmbH}$, ein Teil von Springer Nature 2021

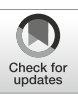

\section{Autor}
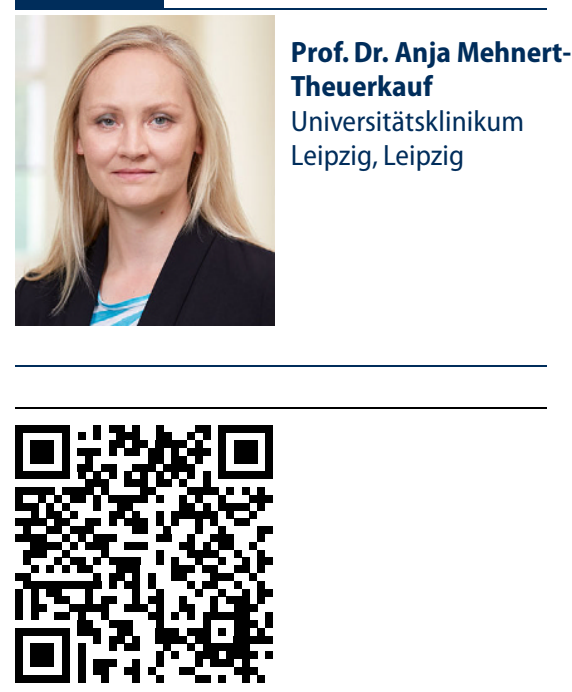

QR-Code scannen \& Beitrag online lesen

\title{
Digitale Tattoos und die Anforderungen an die Krebsmedizin
}

\author{
Anja Mehnert-Theuerkauf \\ Abt. für Medizinische Psychologie und Medizinische Soziologie, Universitätsklinikum Leipzig, Leipzig, \\ Deutschland
}

Liebe Kolleginnen, liebe Kollegen,

in Deutschland entfallen ca. $7 \%$ der 338Mrd. Krankheitskosten auf Krebserkrankungen [1]. Angesichts des demographischen Wandels in unserer Bevölkerung, der steigenden Bedeutung von Krebs mit einer geschätzten Inzidenz von 19,3 Mio. Patienten weltweit [2] sowie der Fortschritte in der Biomedizin ist eine der dringenden Fragen, wie wir eine qualitativ hochwertige, zunehmend kostenintensive Krebsversorgung für älter werdende, multimorbide Patienten sicherstellen können, wenn weniger Menschen diese Leistungen erbringen werden. Krebs stellt in jeder Hinsicht eine Herausforderung dar: für den einzelnen Patienten und seine Familie, für unsere Gesellschaft und für die Gesundheitspolitik.

Die Herausforderungen einer modernen onkologischen Versorgung liegen in der Prävention und frühzeitigen Diagnostik, der Therapie und dem Monitoring der Langzeit- und Spätfolgen der Erkrankung. Damit eng verbunden ist die Digitalisierung in der Medizin. Künstliche Intelligenz (KI) wird eine zunehmend wichtige Rolle dabei spielen, u.a. Routinedaten auszuwerten, individuelle Charakteristiken und Krankheitsverläufe zu erkennen oder vorherzusagen. Dabei sind KI-Algorithmen schnell und erstaunlich zuverlässig. Mit der Notwendigkeit der Digitalisierung sind aber nicht nur Fragen der Datengewinnung verbunden, sondern auch Fragen des Daten- und Patientenschutzes sowie der Datenspeicherung und des Zugangs zu den anfallenden Datenfluten.

Ein besseres Verstehen der genetischen und lebensstilbedingten Ursachen von Krebs, die Vorbeugung und Verringerung der gesundheitlichen Risiken einer Krebserkrankung und die frühzeitige Diagnostik nehmen nach wie vor einen hohen Stellenwert ein. Dies erfordert nicht nur präzisere und spezifischere Krebsbiomarker unterstützt durch besseres Screening, (neue) Diagnosetechnologien wie „liquid biopsy" oder Kl-gestützte Technologien u.a. im Rahmen der onkologischen Bildgebung, sondern auch Krebszentren, die die Gesundheit ihrer Patienten fördern. Gesundheitsförderung benötigt aber auch proaktive Patienten mit guten Gesundheits- und Selbstmanagementkompetenzen.

Im Rahmen des breiten Spektrums der heute verfügbaren Krebstherapien hilft die Präzisionsonkologie, die verfügbaren zielgerichteten Therapien zu identifizieren. Die molekulare Krebsdiagnostik, die mithilfe KI-basierter Algorithmen auf der Grundlage des individuellen molekulargenetischen Profils eines Patienten die richtigen zielgerichteten Therapien identifiziert, ist bspw. ein vielversprechender, aber kostenintensiver Ansatz. Auf diese Weise können Patienten genau die Behandlung erhalten, die für die Art des Krebsgewebes spezifisch ist.

Veränderungen in der onkologischen Versorgung betreffen aber auch das Monitoring und die längerfristige Begleitung von Patienten [3]. Mobile Gesundheitstechnologien wie tragbare oder implantierte Sensoren, digitale "smart" Tattoos, mobile Apps, soziale Medien und Technologien zur Standortbestimmung haben für die Prävention, Diagnostik, Behandlung und Überwachung von Krankheitszuständen bzw. Symptomen sowie für das Wohl- 
befinden und die Lebensqualität von Patienten eine zunehmende Relevanz. Darüber hinaus sind mobile Gesundheitsangebote nicht zuletzt durch die COVID-19-Pandemie mit zunehmender Geschwindigkeit verfügbar.

Voraussetzung für eine patientenzentrierte Versorgung, erfolgreiche Forschung und Translation ist die Verfügbarkeit leistungsfähiger Informationstechnik und leistungsfähiger Infrastrukturen, die auch der Patientensicherheit Rechnung tragen. Die wissenschaftlich und für die Versorgung nutzbaren Daten wachsen exponentiell an, erfordern ein systematisches und einrichtungsübergreifendes Management und damit eine Kultur der Zusammenarbeit.

Ich wünsche Ihnen viel Freude und neue Erkenntnisse beim Lesen der interessanten und anregenden Beiträge dieses Heftes zum Thema Krebs und Politik.

Ihre

Anja Mehnert-Theuerkauf

\section{Korrespondenzadresse}

Prof. Dr. Anja Mehnert-Theuerkauf

Abt. für Medizinische Psychologie und Medizinische Soziologie, Universitätsklinikum Leipzig

Philipp-Rosenthal-Straße 55, 04103 Leipzig, Deutschland

anja.mehnert@medizin.uni-leipzig.de

Interessenkonflikt. A. Mehnert-Theuerkauf gibt an, dass kein Interessenkonflikt besteht.

\section{Literatur}

1. Gesundheitsberichterstattung des Bundes (2021) https://www.gbe-bund.de/ gbe/pkg_olap_tables.prc_set_hierlevel?p_ uid=gast\&p_aid=30470704\&p_sprache=D\&p_ help $=2 \& p \_i n d n r=61 \& p \_a n s n r=62876660 \& p$ version $=2 \& p \_d i m=D .003 \& p \_d w=1000004 \& p$ direction=rollup. Zugegriffen: 22 .Juni 2021

2. Ferlay J, Colombet M, Soerjomataram I et al (2021) Cancer statistics for the year 2020: an overview. Int J Cancer. https://doi.org/10.1002/ijc.33588 (in press)

3. Sim I (2019) Mobile devices and health. N Engl J Med 381(10):956-968

\section{Gefördert durch \\ D Deutsche \\ Forschungsgemeinschaft \\ DGU \\ Deutsche Gesellschaft \\ für Urologie e.V.}

\section{DGU schreibt Teilnahme an der DFG-Nachwuchsakademie "UroAgeCare" aus}

Die neue DFG-Nachwuchsakademie UroAgeCare rückt das Thema "Alter im Kontext von urologischen Erkrankungen und Therapieoptionen" in den Fokus und verfolgt damit das Ziel, durch eine gesteigerte Antragstellung bei der DFG die Forschungstätigkeiten und die daraus resultierenden wissenschaftlichen Erkenntnisse in diesem Bereich der Urologie zu fördern und zu vermehren. Dabei werden junge urologisch forschende Wissenschaftlerinnen und Wissenschaftler in einem frühen Stadium ihrer Karriere auf ihren ersten DFG-Antrag und eine eigenständige Projektleitung herangeführt.

Die Ausschreibung richtet sich an Forscherinnen und Forscher mit medizinischem oder naturwissenschaftlichem Hintergrund, die kurz vor Abschluss ihrer Promotion stehen oder ihre Promotion vor max. 10 Jahren abgeschlossen haben, einer urologischen Klinik oder einem urologischen Forschungslabor angehören oder mit einer solchen Einrichtung assoziiert sind und ein wissenschaftliches Projekt aus dem Themenbereich „Urological Diseases in an Ageing Society - From Genetics to Medical Care" durchführen wollen.

Die Teilnehmerinnen und Teilnehmer der Nachwuchsakademie werden fachspezifisch durch ausgewiesene Wissenschaftlerinnen und Wissenschaftler betreut und sowohl bei der Planung ihrer Forschungsvorhaben als auch bei der Formulierung ihrer DFG-Erstanträge unterstützt. Ein wesentlicher Meilenstein der Nachwuchsakademie ist dabei ein initialer fünftägiger Workshop, in dem sich bis zu 20 Teilnehmerinnen und Teilnehmer mit den Faculty-Mitgliedern und weiteren Gastreferenten zu einem intensiven fachlichen Austausch rund um die wissenschaftliche Agenda von UroAgeCare und die avisierten Projektvorhaben der Teilnehmenden zusammenfinden. Die Ausformulierung der Antragsentwürfe erfolgt dann in einer nächsten Phase, in der die Mentees individuell durch ihre Mentorinnen und Mentoren betreut und beraten werden. Das erste Förderjahr schließt mit der Einreichung der Erstanträge bei der DFG ab.

In der sich anschließenden einjährigen Projektphase erarbeiten die Teilnehmerinnen und Teilnehmer die Grundlagen für eine zweite DFG-Antragstellung - dieses Mal für das normale DFG-Einzelverfahren.

Voraussetzung für die Teilnahme an der Nachwuchsakademie ist die Einreichung einer Projektskizze für ein Forschungsvorhaben mit Beschreibung von Projektidee, Zielsetzung und Grundkonzeption sowie der geplanten Durchführung. Für die Förderwürdigkeit der später bei der DFG einzureichenden (Erst-)Anträge sind vor allem die innovative Idee des Projektentwurfs sowie deren realistische Umsetzbarkeit von Bedeutung.

Interessierte finden alle Informationen zum Bewerbungsverfahren auf der UroAgeCare-Homepage unter https://uroagecare.de

Die Bewerbungsfrist endet am 31. Oktober 2021 


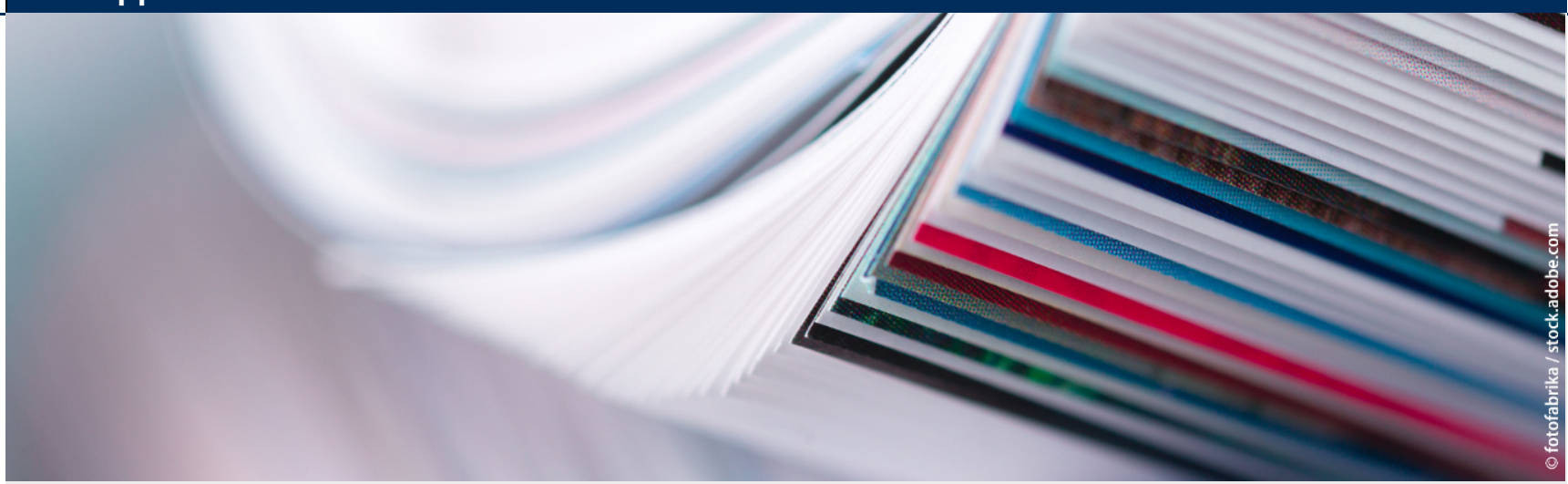

\section{Aktuelle Themenschwerpunkte FORUM - Mitgliedermagazin der DKG}

Diese Leitthemen erwarten Sie in den kommenden Ausgaben

FORUM - bietet Ihnen $6 \mathrm{x}$ jährlich umfassende und aktuelle Beiträge zu interessanten Themenschwerpunkten aus allen Bereichen der Onkologie.

- 01/2021 Chirurgische Onkologie

- 02/2021 Ethik in Onkologie

- 03/2021 "alt, älter, hochbetagt" - Geriatrische Onkologie

- 04/2021 Politik und Krebs

- 05/2021 Kommunikation in der Onkologie

- 06/2021 Krebsforschung in der Onkologie

\section{Ausblick 2022}

- 01/2022 Hot Topic's DKK 2022

- 02/2022 Prävention in der Onkologie

- 03/2022 COVID19 und die Onkologie

- 04/2022 Ethnisch-kulturelle vielfalt in der Onkologie

- 05/2022 Supportivtherapie

- 06/2022 Krebs und Schwangerschaft/Fertilität

(Änderungen vorbehalten)

Alle Inhalte der Zeitschrift finden Sie unter

https://www.springermedizin.de/forum/

SpringerMedizin.de bietet Ihnen Zugang zu allen elektronisch verfügbaren Ausgaben von FORUM vor der Pay Wall, hinter dem Login.

So einfach erhalten Sie Zugang zum Online-Archiv:

- Registrieren Sie sich einmalig auf www.springermedizin.de/register

- Geben Sie Ihre entweder Ihre Einheitliche Fortbildungsnummer (EFN) oder Ihren berufliche Tätigkeit an.

- Ihr Benutzername entspricht Ihrer E-Mail-Adresse, Ihr Passwort können Sie frei wählen und später jederzeit unter "Mein Profil" ändern.

Sind Sie bereits bei SpringerMedizin.de registriert? Haben Sie Probleme bei der Registrierung oder anderen Fragen zum Online-Zugang? Kontaktieren Sie bitte unseren Kundenservice unter: kundenservice@springermedizin.de
Unser Tipp

Mit dem Online-Abo e.Med Interdisziplinär erhalten Sie Zugriff auf alle Fachzeitschriften und die dazugehörigen Fortbildungen. Auf Wunsch erhalten Sie darüber hinaus eine gedruckte Fachzeitschrift Ihrer Wahl aus einem Fachgebiet Ihrer Wahl.

Testen Sie e.Med Interdisziplinär 30 Tage lang kostenlos und unverbindlich!

Jetzt informieren unter

www.springermedizin.de/eMed oder telefonisch unter 0800-77 80777

(Mo - Fr, 10 bis $17 \mathrm{Uhr}$ )
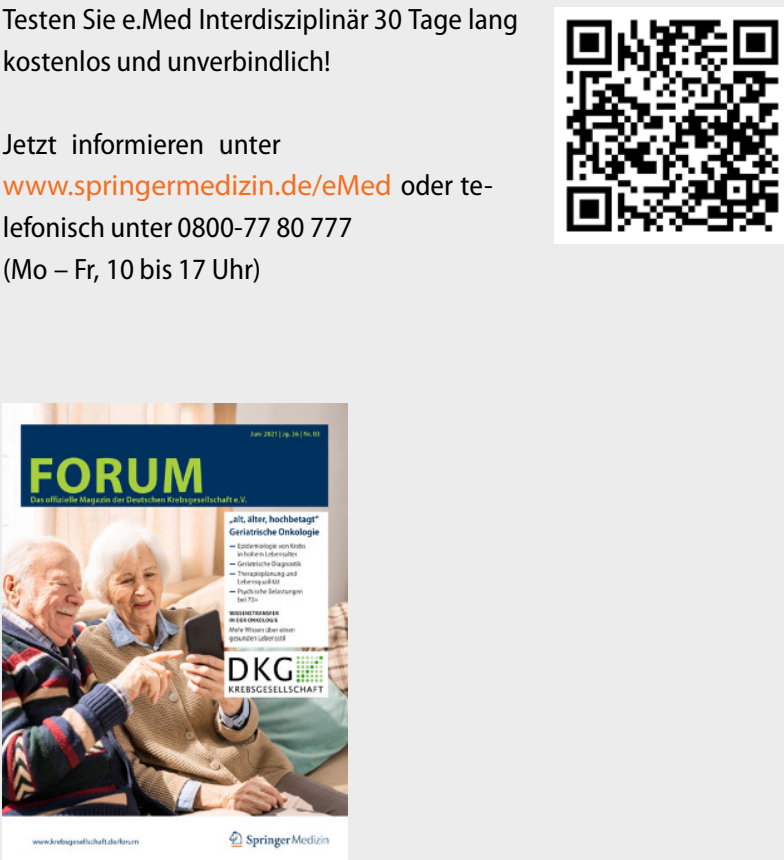